\title{
Desarrollo de la competencia de indagación en Ciencias Naturales
}

\section{Development of the inquiry competence in natural sciences}

Fecha de recepción: 16 de Enero de 2016. Fecha de aprobación: 20 de Octubre de 2017.

Yurany Barrera Cárdenas* Rosalba Cristancho Saavedra **

Artículo de Reflexión

\section{Resumen}

El presente artículo surge del ejercicio de la reflexión basada en el desarrollo de la competencia de indagación en Ciencias Naturales. Es así como se da origen a la creación de un instrumento llamado guía, que direcciona el paso a paso el desarrollo de la competencia de indagación en estudiantes de secundaria de grado noveno de la Institución Educativa Politécnico Álvaro González Santana (IEAGS) de Sogamoso, Boyacá. El colectivo investigador establece los siguientes pasos: observación de fenómenos, planteamiento del problema, formulación de preguntas, formulación de hipótesis y predicciones, búsqueda y registro de información, planteamiento de experimentos e identificación de variables, realización de mediciones y organización de resultados. A propósito de lo anterior, se toman como referencia los parámetros evaluados en la competencia de indagación por el Instituto Colombiano para el Fomento de la Educación Superior (ICFES), todo lo anterior, fundamentado en Aprendizaje Basado en Problemas (ABP). Así pues, el ABP desarrolla progresivamente cada una de las habilidades necesarias para el desarrollo de la competencia indagación y, además, contribuye en la formación del estudiante incrementando el trabajo colaborativo, la responsabilidad y la autoformación.

Palabras clave: indagación ambiental, competencias en Ciencias Naturales, aprendizaje basado en problemas, preguntas investigables, lectura inteligente, educación y medio ambiente.
*Institución Educativa Politécnico Álvaro González Santana - Boyacá - Colombia yyuraa16@yahoo.es

**Institución Educativa

Politécnico Álvaro

González Santana - Boyacá

- Colombia

olgalutty@yahoo.es

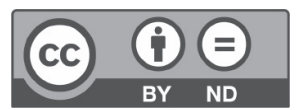




\section{Abstract}

This article arises from the exercise of reflection based on the development of the competence of inquiry in Natural Sciences. This is the origin of the creation of an instrument called a guide, which directs, step by step, the development of inquiry skills in ninth-grade secondary students at the Álvaro González Santana Polytechnic Institution in Sogamoso, Boyacá (IEAGS). The research collective establishes the following steps: observation of phenomena, posing of the problem, formulation of questions, formulation of hypotheses and predictions, search and recording of information, presentation of experiments and identification of variables, measurement and organization of results. With regard to the above, the parameters evaluated in the inquiry competence by the Colombian Institute for the Promotion of Higher Education (ICFES) are taken as reference, all the above, based on Problem Based Learning (PBL). Thus, the PBL progressively develops each of the skills necessary for the development of the inquiry competence and, in addition, contributes to the student's training by increasing collaborative work, responsibility and self-training.

Keywords: environmental inquiry, competences in Natural Sciences, problem-based learning, researchable questions, intelligent reading, education and the environment. 


\section{Introducción}

El diseño de un instrumento modelo que guíe el desarrollo de la competencia de indagación, se hace necesario, debido a las dificultades que presentan los estudiantes observados y a los resultados de las Pruebas Saber de los años 2012 y 2014 de la Institución Educativa Politécnico Álvaro González Santana (IEAGS), en los que se evidencia como debilidad la competencia de indagación en Ciencias Naturales.

Además, en el aula de clase se percibe diariamente que los estudiantes esperan a que se les suministre toda la información que necesitan para el aprendizaje, convirtiéndose en un saber de momento, pues pasados unos días, se hace control de dominio de conceptos y los estudiantes ya nos los recuerdan.

Del mismo modo, se puede identificar que al interior de la comunidad estudiantil es evidente la falta de autoaprendizaje, en donde los saberes se practican por transmisión, y no desde la formación de un espíritu crítico y reflexivo de un saber para qué.

Ahora bien, los parámetros que establece el examen de estado del ICFES, en la competencia de indagación de las Ciencias Naturales, son: observar detenidamente la situación, formular preguntas, recurrir a libros u otras fuentes de información, hacer predicciones, plantear experimentos, identificar variables, realizar mediciones, organizar y evaluar resultados. Para el diseño y aplicación de cada uno de los ítems, se tomaron como guía los conceptos de Melina Furman y María
Eugenia de Podestá en su libro " $\mathrm{La}$ Aventura de Enseñar en Ciencias Naturales" (2014); en ese mismo sentido, se extrae la metodología del ABP publicado por la Universidad de Murcia en España (2008). Este instrumento conduce al estudiante a desarrollar habilidades para: observar detenidamente las situaciones, formular preguntas, buscar relaciones de causa-efecto, recurrir a los libros u otras fuentes de información para seleccionar la más adecuada, y así dar respuesta a las preguntas expresadas teniendo en cuenta su contexto y aplicación.

\section{Conceptos fundamentales}

A continuación, se definen conceptos necesarios para el planteamiento y diseño del instrumento guía en el desarrollo de la competencia de indagación.

Unconceptoeseldecompetencia,entendido como la relación entre conocimientos, habilidades, actitudes, comprensiones y disposiciones cognitivas, metacognitivas, socioafectivas y psicomotoras, que facilitan la eficacia y la eficiencia del sentido de una actividad en un contexto nuevo (Vasco, 2003). Así pues, la competencia en la educación abarca una formación integral, donde se tienen en cuenta todas las dimensiones del ser humano para potencializar sus capacidades.

Otro concepto es indagación, en Colombia es una competencia que se debe desarrollar en el área de Ciencias Naturales en donde se define, según el ICFES (2013), como:

Capacidad para plantear preguntas y procedimientos adecuados para buscar, seleccionar, organizar 
Vale la pena decir que la indagación está muy relacionada con el proceso de investigación, por lo tanto, los estudiantes con el desarrollo de esta competencia desarrollan la capacidad para construir el conocimiento a partir de sus propias experiencias. e interpretar información relevante para dar respuesta a esas preguntas. Por tanto, la indagación en ciencias implica, entre otras cosas, plantear preguntas, hacer predicciones, identificar variables, realizar mediciones, organizar y analizar resultados, plantear conclusiones (p. 101).

Vale la pena decir que la indagación está muy relacionada con el proceso de investigación, por lo tanto, los estudiantes con el desarrollo de esta competencia desarrollan la capacidad para construir el conocimiento a partir de sus propias experiencias. Es así, como un módelo didáctico en la escuela "por indagación podría favorecer el desarrollo en los niños de la habilidad de formular preguntas investigables, una capacidad central al pensamiento científico y que ha sido poco explorada aún” (García \& Furman, 2014, p. 80).

De igual modo, es importante definir el Aprendizaje Basado en Problemas (en adelante $\mathrm{ABP}$ ), el cual se refiere a una estrategia de enseñanza-aprendizaje en el que un grupo de estudiantes (con asesoría de un tutor) se reúne para estudiar, analizar y resolver problemas seleccionados o diseñados para el logro de ciertos objetivos de aprendizaje (Instituto Tecnológico y de Estudios Superiores de Monterrey, s.f., p. 4).

Para llevar a cabo el ABP, se hace necesario un grupo de aprendizaje conformado por el tutor y los estudiantes que asumen roles donde se rota su función. En relación con ello, un estudiante es coordinador y otro secretario; la función del primero, es dirigir la agenda de trabajo con los pasos que se deben llevar a cabo y que se cumplan los tiempos establecidos; la función del segundo, es tomar notas de los acuerdos, registros e información relevante; y, por último, la síntesis de la información (Vizcaro \& Juárez, 2008a).

Adicionalmente, los autores de este artículo idearon un cuarto rol, llamado persona de logística, quien es el encargado de organizar, planear y realizar el montaje de las mediciones o registros que requieran hacerse para el desarrollo del trabajo, sin dejar de lado la participación en la discusión del desarrollo del problema.

También hace parte del grupo el tutor, que en este caso es el profesor, en el que asume un papel de guía y orientador de cada uno de los equipos de estudiantes; por medio de preguntas, orienta la discusión y la exploración permitiendo al estudiante a ser autónomo, reflexivo, siendo esta una guía para el desarrollo del problema y estimular así la creación de conocimiento, olvidando un poco el profesor transmisivo o convencional (Juárez, 2008b).

En esta estrategia, el docente adquiere mayor responsabilidad, ya que la enseñanza y aprendizaje son más personalizados, y dan la oportunidad de utilizar diferentes formas para el desarrollo de cada uno de los problemas, teniendo en cuenta los puntos de vista de los estudiantes.

Por otra parte, un concepto importante es el de "guía de aprendizaje, como herramientaparamotivarintrínsecamente el aprendizaje y suscitar la creatividad 
mediante una cuidadosa selección de sugerencias" (Carrasco, 2004, p. 337) o también definida, como un conjunto de actividades organizadas con una sola finalidad: que el estudiante aprenda, y que aprenda por sí mismo, "acerca de la ejecución de las habilidades..., a utilizar correctamente las estrategias y recursos en el proceso de aprendizaje" (Huertas, Vesga \& Galindo, 2014, p. 59). Además, permite el desarrollo de habilidades, adquisición de conocimientos, actitudes de iniciativa, actitudes de investigación y espíritu crítico (Samaca, 2016, Romero \& Crisol, 2012). Por ende, la escuala se transforma en "un lugar donde los educandos se familiarizan con la lógica de la indagación y su habilidad para investigar, generando de forma sistemática una serie de conocimientos para resolver problemas del contexto"(Acosta, 2013, p. 113).

Por lo tanto, la guía de aprendizaje es una herramienta en la que el docente selecciona, de forma sistemática, una serie de actividades que favorezcan el aprendizaje para que el estudiante ponga en juego su creatividad, y, en este caso, la solución de problemas para el desarrollo de la competencia indagación.

\section{Guía modelo para el desarrollo de la competencia de indagación}

Después de explicar algunos conceptos, se presenta la guía modelo que se diseñó e implementó para el desarrollo de la competencia de indagación en los estudiantes, usando como estrategia el $\mathrm{ABP}$ la cual contiene los siguientes pasos:
Observación del fenómeno. En este paso, el profesor propone observar un gráfico, un dibujo o el proceso de un fenómeno relacionado con el tema a tratar mediantela solución del problema. Lo observado debe ser llamativo y causar impacto en los estudiantes.

La observación del fenómeno debe generar motivación para que despierte la curiosidad y el entusiasmo en el estudiante para proseguir el aprendizaje.

Por consiguiente, el docente debe encaminar el proceso de observación según el objetivo planteado, para que el estudiante haga una descripción detallada haciendo uso de todos sus sentidos; esto, con el propósito de que establezca relaciones entre conceptos y los fenómenos de la naturaleza.

Problema. Un problema es algo que se tiene que resolver y que ignoramos (Restrepo, 2005). Hay que tener en cuenta que, un grupo de profesores de materias afines construyen los problemas (Vizcaro \& Juárez, 2008). Asimismo, es importante pensar detenidamente en cada problema que se va a proponer, para que en el estudio y desarrollo de este se dé el cumplimiento de los objetivos propuestos; no obstante, va dirigido a los estudiantes para que se analice el proceso de indagación para buscar las soluciones posibles.

Según Vizcaro y Juárez (2008), para:

La construcción del problema, se debe decidir: los objetivos de aprendizaje, el tipo de formato en el que se le presentará a los estudiantes como puede ser: relato, video, representación entre otros; también, se debe determinar el
Después de explicar algunos conceptos, se presenta la guía modelo que se diseñó e implementó para el desarrollo de la competencia de indagación en los estudiantes 


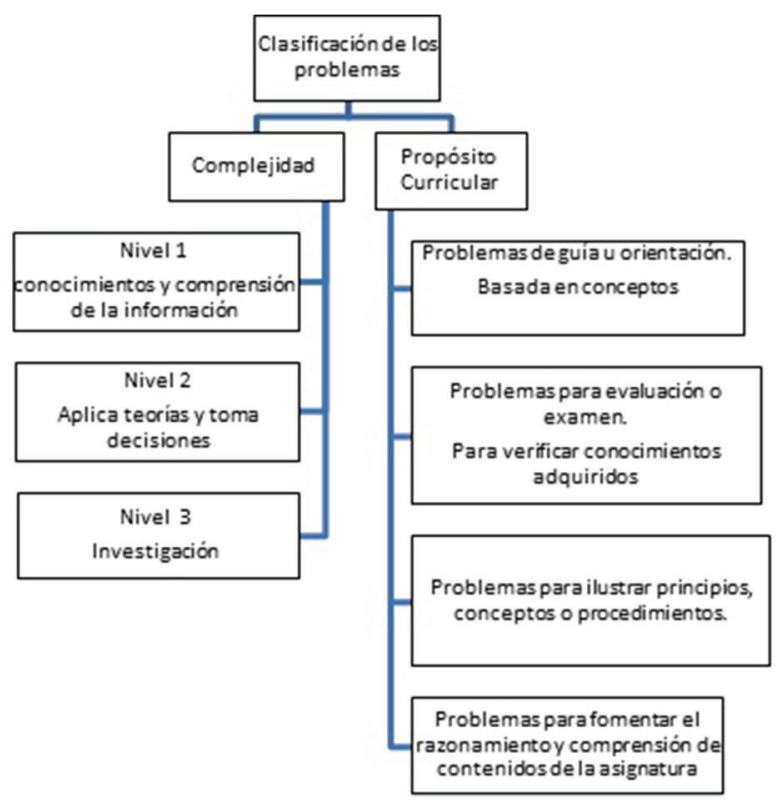

Figura 1. Clasificación de los problemas. Fuente: adaptado de Duch (1996); Duffy \& Cunningham en (1996).

tipo de actividad pertinente para alcanzar los objetivos (p. 20).

En consecuencia, se debe identificar el tipo de problema teniendo algunos parámetros de clasificación (figura 1). De acuerdo con la complejidad, se clasifican en tres niveles (Duch, 1996) que son:

Nivel 1. Solo requiere aplicar conocimientos y comprensión, ya que la información necesaria para resolverlo está dentro del contenido.

Nivel 2. A partir de los conocimientos, el estudiante debe aplicar teoría o tomar decisiones.

Nivel 3. Los estudiantes requieren hacer investigación y desarrollar la capacidad para hacer juicios basados en lo aprendido, el problema no contiene toda la información necesaria para resolverlo, por esto debe buscarse en diferentes fuentes de información (p. 35).

Dado que el modelo fue pensado para el desarrollo de la competencia indagación, el problema planteado se clasifica en el nivel 3, porque se desarrolla como un proceso de investigación para buscar soluciones a los problemas y considera fundamental la selección de información en diferentes fuentes, al igual que analizar críticamente cada uno de los textos, recolección de datos y observaciones para realizar deducciones.

Otra clasificación es la descrita por Duffy \& Cunningham (1996), se relaciona con el propósito curricular y propone cuatro tipos de problemas, que son:

Problemas de guía u orientación. En los conceptos centrales del curso se focaliza la atención del estudiante.

Problemas para evaluación o examen. Aplicación de problemas en un examen de los conocimientos adquiridos.

Problemas para ilustrar principios, conceptos o procedimientos. Son problemas en el que el estudiante descubre procesos, explicaciones $\mathrm{O}$ definiciones, impulsado mediante el método inductivo propuesto por el profesor.

Problemas para fomentar el
razonamiento y comprensión de
contenidos de la asignatura. Las 
habilidades cognitivas de razonamiento, el análisis, y la síntesis de información, se logran estimular mediante este tipo de problemas.

De la anterior clasificación, los problemas planteados en el ABP, son los que fomentan el razonamiento y la comprensión de los contenidos de la asignatura, desarrollando habilidades cognitivas, mediante el análisis de información obtenida de diferentes fuentes y en el que el estudiante alcance un aprendizaje significativo.

Posteriormente, se tienen en cuenta criterios como: la elaboración del problema, contenido del problema y resolución del problema referidos en la metodología ABP (ver figura 2).

\section{La elaboración del problema}

Estructuración. Una de las características del problema es su "mala estructuración", quiere decir que el problema debe tener varias opciones de solución, poca información o datos, y debe ser impreciso, complicados de aclarar (Hallinger \& Bridges, 1996). Por ello, este es un punto primordial, debido a que la redacción debe obedecer a que los estudiantes tengan diferentes caminos para llegar a su solución.

Adicionalmente, deben ser problemas "abiertos", lo cual significa que no todos los componentes del problema deben ser conocidos, y que tengan varias soluciones (Prieto, 2006).

Complejidad. Los problemas deben ser difíciles de resolver y no deben tener

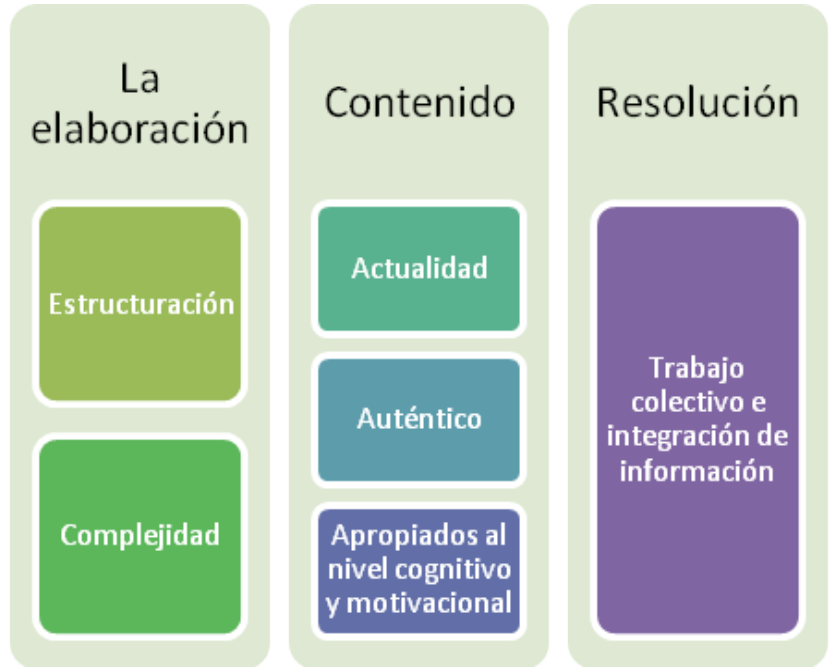

Figura 2. Criterios de la formulación de un buen problema. Fuente: Adaptado de Hallinger \& Bridges, 2007; Stinson \& Miler (1996); Duch, \&Allen, (1998).

una única solución (Duch, 1996). Se considera que, estos deben tener un nivel de dificultad de acuerdo con la edad del estudiante y su dialéctica.

\section{Contenido del problema}

Actualidad de los problemas. Se consigue motivar y enganchar a los estudiantes con problemas de la actualidad o contemporáneos (Stinson \& Miler, 1996). En este caso, se debe contener casos de la vida cotidiana en donde se logre tener la inquietud de los estudiantes por conseguir la realidad.

Auténticos. Relevantes para el alumno o basado en la vida real o profesional. Los problemas deben ser relacionados con la planeación del curso. Por lo tanto, deben ser prácticos y enlazados con la vida cotidiana (Delisle, 1997).

\section{Apropiados al nivel cognitivo $y$ motivacional de los alumnos. Para}

De la anterior clasificación, los problemas planteados en el $A B P$, son los que fomentan el razonamiento y la comprensión de los contenidos de la asignatura, desarrollando habilidades cognitivas, mediante el análisis de información obtenida de diferentes fuentes y en el que el estudiante alcance un aprendizaje significativo. 
La resolución del problema no solo trata de buscar información, sino que en trabajo colectivo se sintetice ideas, se tomen decisiones para resolverlo, integración de la información y comunicación de resultados que toque el interés y la emoción del estudiante, el problema debe estar a su nivel de conocimiento y de desarrollo intelectual (Prieto, 2006).

\section{Resolución del problema}

La resolución del problema no solo trata de buscar información, sino que en trabajo colectivo se sintetice ideas, se tomen decisiones para resolverlo, integración de la información $y$ comunicación de resultados (Duch \& Allen, 1998).

A su vez, el docente puede aplicar la lista de chequeo (ver tabla 1) para comprobar la calidad del problema.

Formula preguntas: el estudiante plantea preguntas con base en las inquietudes que le genere el problema, y que le sirvan de guía para la búsqueda de soluciones.

Frente a eso, las preguntas que plantean los estudiantes pueden ser de cuatro tipos, estas son: las que exclaman sorpresa y manifiestan interés, las preguntas que piden conocimientos, las preguntas que reflexionan sobre la esencia de las cosas naturales, y las preguntas investigables (Harlen, 2007).

Así pues, en la enseñanza de las ciencias y especialmente en el desarrollo de la competencia indagación, se requiere de la formulación de diferentes tipos de preguntas, pero el tipo de pregunta imprescindible es la investigable que se debe acompañar de preguntas que involucren la búsqueda de información (ver figura 3).

A continuación, se muestran ejemplos de preguntas de diferentes tipos, clasificadas de acuerdo con lo que se busca obtener en la respuesta (Roca, Márquez \& Sanmartí, 2013).

Preguntas que orientan la búsqueda de conceptos y conocimientos, estas

Tabla 1.

Lista de comprobación (checklist) de la calidad de un problema ABP.

\begin{tabular}{|c|c|c|c|c|}
\hline \multicolumn{2}{|c|}{ Ítems de comprobación del diseño del problema } & Sí & No & Notas \\
\hline \multirow{3}{*}{$\begin{array}{lr}\text { El problema } & \text { puede } \\
\text { motivar a los } \\
\text { estudiantes y } & \text { ser } \\
\text { atractivo para ellos por } \\
\text { ser una cuestión: }\end{array}$} & 1.1. Social & & & \\
\hline & 1.2. De la vida diaria & & & \\
\hline & 1.3. De las asignaturas & & & \\
\hline \multicolumn{2}{|c|}{ El problema refleja una situación actual. } & & & \\
\hline \multicolumn{2}{|c|}{ Cubre objetivos didácticos de la asignatura o curso. } & & & \\
\hline \multicolumn{2}{|c|}{ Es apropiado al nivel cognitivo y motivacional de los alumnos. } & & & \\
\hline \multicolumn{2}{|c|}{ El problema está mal estructurado. } & & & \\
\hline \multicolumn{2}{|c|}{$\begin{array}{l}\text { El problema es complejo y, por tanto, requerirá colaboración } \\
\text { grupal para su solución. }\end{array}$} & & & \\
\hline \multicolumn{2}{|c|}{ El problema se puede desarrollar basado en la indagación. } & & & \\
\hline
\end{tabular}

Fuente: adaptado de Romero \& García (2008). 


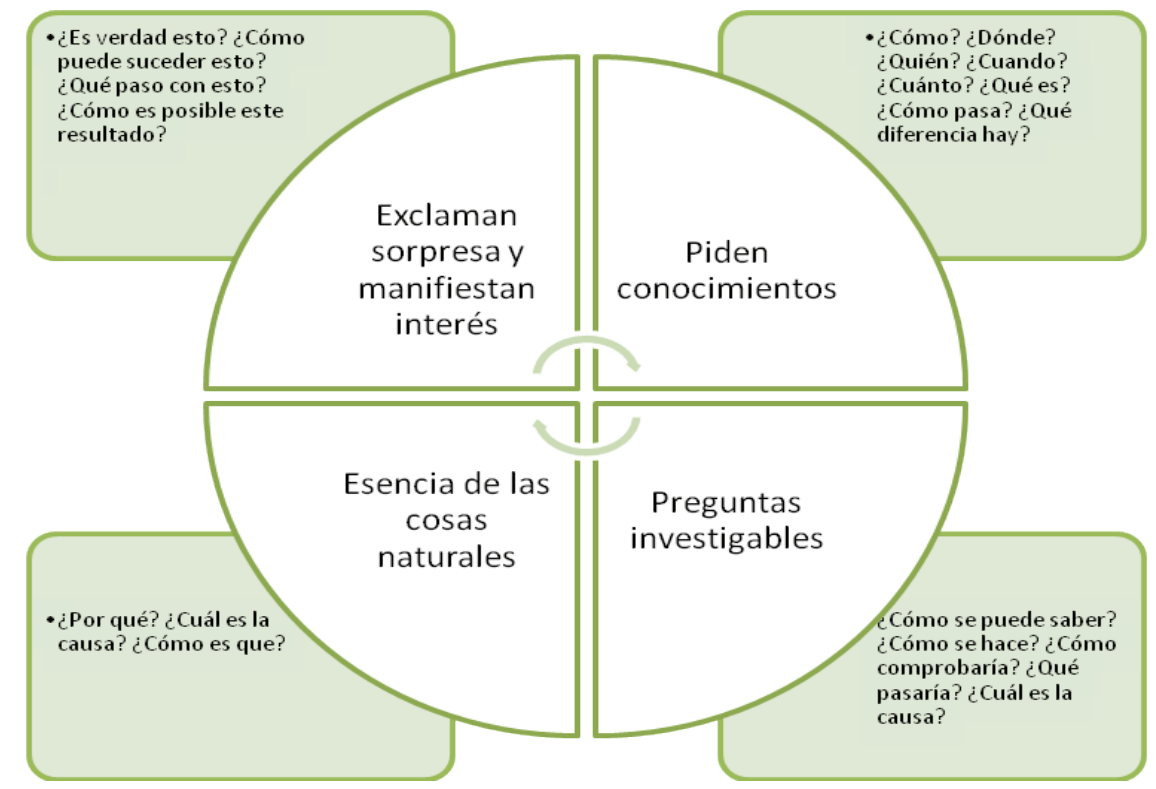

Figura 3. Tipos de preguntas.

Fuente: adaptado de Roca, Márquez \& Sanmartí (2013).

dan cuenta de fenómenos, procesos o conceptos. Como ejemplo, tenemos: ¿Cómo?, ¿Dónde?, ¿Quién?, ¿Cuándo?, ¿Cuánto?, ¿Qué es?, ¿Cómo pasa? y ¿Qué diferencia hay?

Preguntas orientadas a explicar. Ejemplo: ¿Por qué?, ¿Cuál es la causa? y ¿Cómo es que?

Preguntas orientadas a la investigación. Ejemplo: ¿Cómo se puede saber?, ¿Cómo se hace?, ¿Cómo comprobaría?, ¿Qué pasaría? y ¿Cuál es la causa?

Realiza predicciones o formula hipótesis. La explicación tentativa de un fenómeno que puedan eventualmente ser puestos a prueba y que debe generar predicciones, es una hipótesis (Furman \& de Podestá, 2014). En esta formulación de hipótesis y predicciones, es importante que el profesor induzca a los estudiantes para que den explicaciones de los fenómenos a partir de su experiencia que les permitan ir más allá de lo superficial que ofrece el mundo, en el cual ponga a prueba sus sentidos para hacer de cada situación y vivencia una construcción de saber, que hará parte de la humanidad (Cañizalez \& Pulido, 2015). Igualmente, para que en los grupos se discuta las posibles explicaciones y así determinar cuál es la más coherente. Además, debe fomentar la búsqueda de información en diferentes fuentes, de tal manera que se sumen estas ideas a las suyas y las de sus compañeros (Harlen, 2007).

Después de hacer la observación del fenómeno, de analizar el problema, de formular preguntas, especialmente de tipo investigable; ahora, corresponde dar posibles explicaciones al porqué suceden los hechos y las hipótesis que pueden ser susceptibles a la comprobación.
La explicación tentativa de un fenómeno que puedan eventualmente ser puestos a prueba y que debe generar predicciones, es una hipótesis (Furman \& de Podestá, 2014). 
El estudiante consulta y diseña experiencias en las que pueda poner a prueba su hipótesis, siempre teniendo cuidado de explicar el porqué de cada paso de la experimentación
Al respecto, es importante decir que, hay diferencia entre predicción y suposición. La primera se basa en teorías o conceptos; mientras que la segunda es una idea que se da por cierta o verdadera sin ninguna argumentación o fundamentación teórica.

Búsqueda y registro de información: el estudiante necesita desarrollar la capacidad no solo de buscar información, sino de seleccionarla e interpretarla (Pozo \& Gómez, 2006).

Al mismo tiempo, el estudiante debe disponer de diferentes fuentes de información que le permitan aclarar los conceptos, teorías y, en general, los conocimientos acerca del tema para solucionar el problema; posteriormente, de tener la información, debe ser capaz de seleccionar la que verdaderamente es necesaria; $y$, además, de organizarla de tal forma que haga una lectura inteligente.

Cabe señalar que, la lectura inteligente es la capacidad de hacer una lectura comprensiva que permita aplicar, analizar, hacer síntesis, comparar, memorizar, parafrasear, valorar, constatar, inferenciar, seleccionar, utilizar, proyectar y crear (Nuñez, 2011).

Con respecto a este paso, los investigadores establecen que el secretario es el encargado de registrar la información, resultado de la consulta, el análisis y la discusión establecida en el grupo sobre el problema.

Sumado a lo expuesto, cada grupo debe llevar su registro en un portafolio en el que se evidencie el progreso en la investigación, hasta obtener la solución o las posibles soluciones.

Plantea experimentos e identifica variables: el estudiante consulta y diseña experiencias en las que pueda poner a prueba su hipótesis, siempre teniendo cuidado de explicar el porqué de cada paso de la experimentación, para encontrarle lógica y relacionarlo con la hipótesis y, por supuesto, con la solución del problema, es así como, "La rutina diaria de cualquier persona implica la adquisición y el manejo de habilidades que le permitan agilizar las diferentes actividades que la componen" (Pachón, Parada \& Zamir, 2016, p. 224) resolviendo un problema. Es preciso hacer énfasis en la importancia de dedicarle el tiempo suficiente para analizar las observaciones del proceso y de los resultados de las experiencias, de tal forma que identifique las variables que afectan el comportamiento del fenómeno y, si es necesario, repetir las experiencias.

Realiza mediciones y toma datos de las mediciones: el estudiante en el proceso de la experimentación, identifica las variables que afectan el fenómeno, diseña instrumentos para registrar los datos de las mediciones o de los resultados; en muchos casos, se deben establecer tablas y graficar, de tal forma que se facilite su análisis e interpretación para así deducir conclusiones.

\section{Organiza resultados y los sustenta:} organiza los resultados, no solo de la parte experimental sino de todo el proceso relacionando cada una de las etapas con los conocimientos y teorías 
para deducir el cumplimiento o no de la hipótesis planteada.

Además, se debe correlacionar los resultados con el problema planteado para verificar si fue posible solucionarlo o retomar el proceso. Cuando se identifica que ya se encontró la solución al problema, se organiza toda la información tanto del proceso, como de los conocimientos y los resultados, en forma clara, para ser socializada a todos los integrantes del curso y exponer así la conclusión final.

\section{Aplicación de la guía}

Antes del inicio a la aplicación de la guía, se debe realizar una explicación del paso a paso a realizarse en cada uno de las etapas anteriormente expuestas, con el fin de tener mayor claridad en el procedimiento que el estudiante va a realizar.

Así pues, la guía debe concordar con el nivel cognitivo y motivacional del estudiante, para generar en él mayor interés en su desarrollo.

Por otro lado, se debe desarrollar en varias sesiones de dos horas cada una, debido a que si al estudiante se la aplican jornadas más largas, se genera cansancio y monotonía en el desarrollo de esta, observándose la disminución en el interés.

Así mismo, se debe estipular el tiempo para la finalización del proceso, debido a la necesidad de dar cumplimiento al planeamiento de la asignatura, para evitar quedar estancados en una sola temática, con lo cual no se lograría cumplir con los estándares establecidos para el nivel correspondiente; y, aparte de ello, hay que incentivar la responsabilidad para el desarrollo del paso a paso de la guía y evitar los tiempos muertos (distracción).

Igualmente, el tutor tiene que estar en continua revisión por cada uno de los equipos conformados por los estudiantes, con el objetivo de orientar el proceso y desarrollo de la guía.

Del mismo modo, se recomienda que el sitio de trabajo sea cómodo, que brinde satisfacción y cumpla las necesidades de los estudiantes para el trabajo a realizar.

\section{Resultados de la aplicación}

Al aplicar la secuencia de guías, se observó un progreso en la capacidad de los estudiantes para diseñar preguntas que generen investigación, describir observaciones, hacer predicciones, elegir la información necesaria para solucionar el problema, desarrollar prácticas para la comprobación de su hipótesis, elegir instrumentos adecuados para registrar los datos obtenidos y luego los representa en gráficos y tablas, los cuales le facilitan la interpretación de los datos resultantes y elaborar conclusiones.

Sin embargo, se detectan dificultades en el momento de comunicar la información sobre el proceso y solución del problema. Lo anterior se presenta debido al poco tiempo para el desarrollo de las últimas etapas, y a que en pocas oportunidades a los estudiantes se les permite la
Antes del inicio a la aplicación de la guía, se debe realizar una explicación del paso a paso a realizarse en cada uno de las etapas anteriormente expuestas, con el fin de tener mayor claridad en el procedimiento que el estudiante va a realizar. 


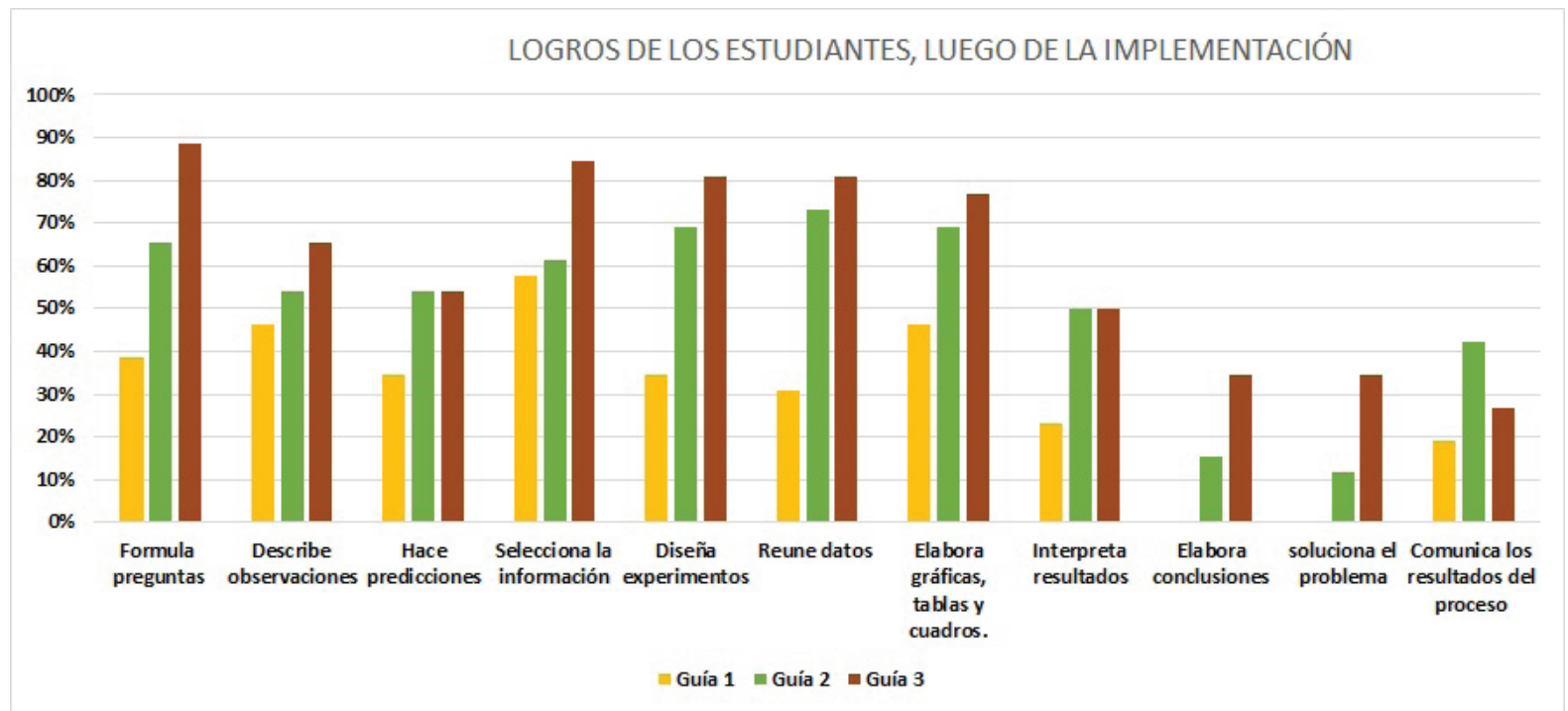

Figura 4. Resultado de guías implementadas.

comunicación oral de los resultados de sus trabajos, es así que les falta fluidez y coherencia en la socialización de los resultados.

Por otra parte, los procesos que lograron mayores avances porque superaron el $80 \%$, fueron: preguntas que generan investigación, la elección de información pertinente de diferentes fuentes, el desarrollo de experimentos que permiten analizar el problema y elige las herramientas necesarias para reunir datos del proceso de experimentación.

Cabe aclarar que, el estudiante en la etapa de describir observaciones, por ser una etapa inicial del proceso, requiere de sus conocimientos previos para poder hacer una descripción detallada, y no depende únicamente de la habilidad para describir.

Además, los procesos en los que se obtuvieron los porcentajes más bajos, fueron: hacer predicciones, análisis e interpretación de resultados, elaborar conclusiones y la socialización de resultados, se detectó que los estudiantes al no tener el conocimiento previo necesario escribían ideas y no predicciones. A pesar de las mejoras en la interpretación y la elaboración de conclusiones, se evidencia que estos procesos requieren de mayor trabajo y tiempo, más cuando el estudiante no está acostumbrado a esta metodología que requiere autonomía y autoaprendizaje.

Conviene subrayar que, los estudiantes que lograron solucionar el problema propuesto por el docente, están enlazados a los que logran analizar datos y establecer conclusiones.

Como el problema es la base del ABP, se percibe que este motiva al estudiante para descubrir su solución, y es la base que guía al estudiante para el desarrollo de las etapas del proceso a seguir. 


\section{Conclusiones}

Expuesta la estructura de la guía y la forma de desarrollarla, los investigadores describen las ventajas y logros de su implementación.

Al analizar el modelo de guía propuesto en la investigación por los autores y solucionado por los estudiantes del grado 903 (química) y 904 (física) del colegio politécnico "AGS" de Sogamoso, se evidencia el desarrollo de la competencia indagación, ya que los estudiantes generan las habilidades y desempeño en plantear preguntas de tipo investigable, seleccionar la información pertinente para la solución del problema, proponer experimentos para dar respuesta a sus preguntas, representar datos en gráficas, tablas y cuadros, generar conclusiones a partir de los resultados siendo estos algunos de los parámetros establecidos por el ICFES (2015) para la competencia de indagación.

Además, se genera un cambio de rol del estudiante y del docente al implementar la estrategia, puesto que el estudiante toma el rol activo, es quien guía el proceso de construcción de su conocimiento y los compromete con su aprendizaje; y el docente es el orientador que acompaña y motiva durante todo el proceso, para evitar que el estudiante pierda de vista la meta a alcanzar.

Adicionalmente, los autores comprobaron que los problemas planteados cumplieran con las siguientes características: son de la vida cotidiana, está de acuerdo con el nivel cognitivo de los estudiantes, es abierto (se puede solucionar de diferentes maneras), necesita del trabajo colaborativo, que el $A B P$ es una excelente estrategia para generar en el estudiante gran curiosidad, estímulo por la búsqueda de soluciones de problemas, dando fundamento al desarrollo de la competencia indagación.

Por otra parte, el estudiante al apropiarse de su aprendizaje, lo obliga a aprender a aprender, por lo tanto, los conocimientos son mucho más profundos, completos y de carácter significativo.

De la misma forma, en el aspecto social se observa el desarrollo de un gran número de valores que se generan en el estudiante, tales como: seguridad, confianza, liderazgo, tolerancia, facilidad para el trabajo en equipo, entre otras.

Por ende, al hacer un trabajo colaborativo entre los integrantes del equipo, hace que entre estudiantes y el docente se creen lazos de amistad más cercanos y de confianza, el cual es un ambiente ideal de aprendizaje.

En efecto, el desarrollo de cada etapa es muy importante para poder alcanzar el desarrollo de la competencia indagación, así pues, se debe enfatizar en la lectura y análisis del problema planteado por parte de los estudiantes para que así ellos puedan determinar cuáles serán las preguntas que guiarán la búsqueda de la solución del problema.

Al analizar el modelo
de guía propuesto en
la investigación por los
autores y solucionado por
los estudiantes del grado
903 (química) y 904 (física)
del colegio politécnico
"AGS” de Sogamoso, se
evidencia el desarrollo
de la competencia
indagación, ya que los
estudiantes generan las
habilidades y desempeño
en plantear preguntas de
tipo investigable




\section{Referencias}

Acosta Muñoz, J. (2013). Las actitudes investigativas en la formación escolar. Praxis \& Saber, 4(8), 109-133. doi:https://doi.org/10.19053/22160159.2654

Carrasco, J. B. (2004). Una didáctica para hoy cómo enseñar mejor. Madrid: Ediciones RIALP, S.A.

Cañizalez, N., \& Pulido, O. (2015). Infancia, una experiencia filosófica en el cine. Praxis \& Saber, 6(11), 245-262. doi:https://doi. org/10.19053/22160159.3583

Delisle, R. (1997). How to Use Problem-Based Learning in the Classroom. Recuperado el 15 de agosto de 2016, de http://www.ascd.org/publications/books/197166/ chapters/Developing-a-Problem.aspx

Dirección de Investigación y Desarrollo Educativo, Vicerrectoría Académica, Instituto Tecnológico y de Estudios Superiores de Monterrey. (s.f.). El Aprendizaje Basado en Problemas como técnica didáctica. Recuperado de http:// www2.uca.es/ordenacion/formacion/docs/jifpev4-documentacion.pdf

Duch, B. (1996). Problems: A Key Factor in PBL. Recuperado de https://www1.udel. edu/pbl/cte/spr96-phys.html

Duch, B., \& Allen, D. (1998). Problem-based learning: Preparing students to succeed in the 21st century. Recuperado el 8 de junio de 2016, de http:/ocw.um.es/ cc.-sociales/la-metodologia-de-aprendizaje-basado-en-problemas/material-declase-1/tema-4.pdf

Duffy, T. M., \& Cunningham, D. J. (1996). Constructivismo: Implicaciones para el diseño $y$ la entrega de la instrucción. New York: INDIANA UNIVERSITY.

Furman, M., \& PodestÁ, M. DE (2014). La aventura de enseñar Ciencias Naturales. Buenos Aires: AIQUE educación.

García, S., \& Furman, M. (2014). Categorización de preguntas formuladas antes y después de la enseñanza por indagación. Praxis \& Saber, 5(10), 75-91. doi:https://doi.org/10.19053/22160159.3023 Restrepo GoméZ, B. (2005). Aprendizaje basado en problemas (ABP): una innovación didáctica para la enseñanza universitaria. Educación y Educadores, 8, 9-19. Recuperado de http:// www.redalyc.org/articulo.oa?id=83400803

Hallinger, P, \& Bridges, (2007). Problem-based learning: a promising approach to management education. In: A Problem-based Approach for Management Education. Springer, Dordrecht. doi: 10.1007/978-1-4020-5756-4

HARlen, W. (2007). Enseñanza y aprendizaje de las ciencias. Madrid: Ediciones Morata, S.L.

Huertas, A., Vesga, G., \& Galindo, M. (2014). Validación del instrumento 'inventario de habilidades metacognitivas (Mai)' con estudiantes colombianos. Praxis \& Saber, 5(10), 55-74. doi:https://doi.org/10.19053/22160159.3022 
ICFES. (Febrero de 2015). Lineamientos generales para la presentación del examen de Estado SABER $11^{\circ}$. GUÍAS Sistema Nacional de Evaluación Estandarizada de la Educación. Bogotá: Ministerio de Educación.

ICFES. (2013). Sistema Nacional de Evaluación Estandarizada de la Educación Alineación del examen SABER 11․ Alineación del examen SABER 11, 101.

JuÁrez, C. V. (2008a). ¿Qué es y cómo funciona el aprendizaje basado en problemas? En U. d. Murcia, La Metodología del Aprendizaje por Problemas (p. 15). España: Servicio de Publicaciones.

JuÁrez, C. V. (2008b). La metodología del aprendizaje basado en problemas. Madrid: Servicio de publicaciones.

NuñEz, A. E. (2011). Didáctica de la Lectura eficiente. México: Universidad Autónoma del estado de México.

Pachón, L., Parada, R., \& Chaparro, A. (2016). El razonamiento como eje transversal en la construcción del pensamiento lógico. Praxis \& Saber, 7(14), 219-243. doi:https://doi.org/10.19053/22160159.5224

Pozo, M. J. I., \& Gómez, C. M. A. (2006). Aprender y enseñar ciencias del conocimiento cotidiano al conocimiento cientifico. Madrid: Ediciones Morata, S.L.

Prieto, M. A. (2006). ICE Universidad de oviedo. Recuperado el 12 de agosto de 2016, de: http://www.habilidadesparaadolescentes.com/archivos/Articulo_ ABP_y_desarrollo_de_competencias.pdf.

Roca, T. M., Márquez, C., \& Sanmartí, N. (2013). Las preguntas de los alumnos: Una propuesta de análisis. ENSEÑ ANZA DE LAS CIENCLAS. Revista de investigación y experiencias didácticas, 31(1), 95-114.

Romero, M. A., \& Crisol, M. E. (2012). Las guias de aprendizaje autónomo como herramienta didáctica de apoyo a la docencia, 15, 9-31. Recuperado de http://www. ceuandalucia.es/escuelaabierta/pdf/articulos_ea15pdf/romero_ea15.pdf

Romero, M. A., \& García, S. J. (2008). La elaboración de problemas ABP. En J. García Sevilla, El aprendizaje basado en problemas en la enseñanza universitaria ( $\mathrm{p}$. 53). Madrid: Universidad de Murcia, Servicio de Publicaciones.

SamacÁ, I. (2016). El espíritu científico en la primera infancia. Praxis \& Saber, 7(13), 89-106. doi:https://doi.org/10.19053/22160159.4167.

Stinson, J. E., \& Miller, R. G. (1996). Problem-Based Learning in Business Education: Curriculum Design and Implementation Issues. Recuperado el 13 de agosto de 2016, de http://onlinelibrary.wiley.com/doi/10.1002/tl.37219966807/epdf?r3_ referer=wol\&tracking_action=preview_click\&show_checkout=1\&purchase _ referrer=www.google.com.co\&purchase_site_license=LICENSE_DENIED

VAsco, C. E. (2003). Objetivos específicos, indicadores de logros y competencias ¿y ahora estándares? Educación y Cultura, 33-41. 